\title{
SOBRE LA IMPOSIBILIDAD DE UN PRIMER PRINCIPIO ABSOLUTO DE LA FILOSOFÍA (1795) ${ }^{1}$
}

\author{
ON THE IMPOSSIBILITY OF A FIRST ABSOLUTE PRINCIPLE \\ OF PHILOSOPHY (1795)
}

Paul Johann Anselm Feuerbach

La tarea más urgente de la razón filosófica desde la gran revolución de la filosofía operada por el criticismo se orienta a encontrar un principio supremo de toda la filosofía; un principio que sea absoluto, que esté fundamentado únicamente en sí mismo, y que sea el primer eslabón en la cadena del saber filosófico, o incluso - como algunos pretenden — de todos los conocimientos humanos. — Una tarea muy respetable, pero que ya a primera vista parece abarcar tanto, que de entrada debemos poner en duda, con una justificada desconfianza hacia las fuerzas de la razón humana, que pueda llevarse a cabo felizmente; y como la posibilidad de un principio tal no es evidente por sí misma, debemos plantearnos la siguiente pregunta: ¿es acaso posible ballar un principio supremo, absoluto, no necesitado de ninguna prueba ulterior?, a fin de asegurarle a la razón la legitimidad de su procedimiento, o bien poner freno a su presunción, si la pregunta debiera responderse negativamente.

La respuesta a esta pregunta es el objeto de este ensayo, que en modo alguno pretende dirimirla definitivamente, sino que debe ser considerado como el dictamen sin pretensiones de un particular.

El sentido común está en posesión de ciertas condiciones que están inmediatamente unidas al interés del corazón humano y de las que no puede renunciar sin renegar de sí mismo. Estas convicciones atañen a la virtud, el derecho, la divinidady la inmortalidad. Acerca de estos asuntos el sentido común de los hombres es enteramente unánime, y el hombre podría conformarse con estas sentencias inmediatas de su corazón — quedando igual todo lo demás—, si no fuese porque la razón misma le perturba en la tranquila posesión de esta convicción y le obliga a abandonar la tierra de los sentimientos. La razón no puede conformarse con

\footnotetext{
1 “Über die Unmöglichkeit eines ersten absoluten Grundsatzes der Philosophie" Philosophisches Jahrbuch II/4 (1795), pp. 306-322. Traducción de José Luis López de Lizaga.
} 
meros sentimientos, ella tiene que exigir una convicción basada en razones. En virtud de su naturaleza, está obligada a poner en cuestión estos sentimientos, dudar de la realidad de los juicios fundados en ellos, y declararlos ficticios mientras no se haya encontrado una prueba estricta de ellos. Ahora bien, si el espíritu humano no quiere caer en contradicción consigo mismo, debe transferir la justificación de estos sentimientos precisamente a la razón que niega su realidad y que, en la medida en que tiene por objetivo la solución de estos problemas, se llama razón filosofante. ${ }^{2}$

Pero la solución de dichos problemas es demasiado difícil, las armas posibles o reales del escepticismo contra estos sentimientos son demasiado poderosas, y el interés del corazón humano está demasiado vinculado a esas convicciones, como para que la razón pudiese alcanzar su objetivo sin grandes pertrechos. Estos pertrechos, sin los cuales la razón no puede dar en absoluto una respuesta completamente satisfactoria a esos problemas del sentido común, consisten nada menos que en la exploración completa de la totalidad de la mente humana, pues sólo así la razón está en condiciones de proteger el paladión de la humanidad contra los ataques de la duda.

La ciencia cuya finalidad suprema debería ser responder a esos problemas, y cuyo objeto debería ser el espíritu humano, es la filosofía.

Ahora bien, ¿mediante qué principium cognoscendi alcanzamos un conocimiento en filosofía? - El conocimiento general sólo es posible por dos vías, o bien a partir de la experiencia a posteriori, o bien a partir de aquello que precede a toda experiencia, a priori: y por eso la filosofía se divide, por lo que respecta a su modo de conocimiento, en filosofía empirica y filosofía pura. Es empírica cuando obtiene su materia de la experiencia, y es pura cuando obtiene su materia no de la experiencia, sino a priori.

Pero ¿cómo es posible la filosofía empírica como ciencia? - No nos referimos a cómo alcanza la filosofía empírica su material, pues la respuesta a esta pregunta ya está dada por la mera expresión "filosofía empírica". Más bien nos preguntamos cómo el material dado empíricamente puede convertirse en ciencia, o mejor: puede adquirir la forma de la ciencia. — La ciencia es una totalidad de

\footnotetext{
2 "Buscar razones de las cosas", como cree Jakob (en su Lógica); o "poner unidad en lo diverso de los conocimientos", como dice Maimon (vid. Bacon, Neues Organon, traducción de Bartoldy. Prólogo, p. LXVIII), no significa todavía filosofar sino pensar. El pensamiento filosófico es un tipo especial de pensamiento y sólo puede distinguirse del pensamiento en general por el objetivo al que se dirige; y este objetivo no es otro que la solución de los problemas prácticos del sentido común.
} 
conocimientos diversos reunidos formando una unidad. Pero por eso lo diverso de los conocimientos empíricos debe ser reunido. Esto sucede ya mediante el orden de las partes, o con otras palabras: mediante la forma sistemática. Pero la razón no puede conformarse con esta composición mediante la mera ordenación de las partes, sino que exige además una conexión de los conocimientos, y esto sólo es posible mediante una conexión causal, y ésta sólo es posible mediante fundamentos [Gründe]. Pero los fundamentos son o bien fundamentos de conocimiento, o bien fundamentos explicativos. Los fundamentos de conocimiento son tales que determinan los conocimientos mismos sólo mediante los cuales alcanzamos un conocimiento; pero los fundamentos explicativos son tales que no determinan el conocimiento mismo, sino sólo la existencia del objeto de conocimiento, es decir: contienen el fundamento por el cual el objeto como tal resulta conocido. - Ahora bien, las proposiciones de la filosofía empírica tienen la experiencia como principio, ella es el fundamento del conocimiento, y lo que se basa en este principio es inmediatamente cierto y no precisa de un conocimiento mediato a partir de fundamentos. Por eso los fundamentos de la filosofía empírica deben ser fundamentos explicativos, y la tarea del filósofo empírico en la construcción de su ciencia consiste en indagar las leyes de la naturaleza humana, elevarse de los datos particulares de la experiencia a las leyes generales, y descender con su ciencia de nuevo desde estas leyes y explicar los datos subordinados de la mente humana.

Muy distintas son las cosas en el caso de la filosofía pura. Aquí la experiencia nos abandona completamente. Por tanto, se plantea la cuestión siguiente: ¿cómo es posible la filosofía pura, no sólo con respecto a su forma, sino también con respecto a su materia?

Como en el terreno de la filosofía pura he sido abandonado por la experiencia, pero sólo la experiencia garantiza un conocimiento inmediato, de esto se sigue que la materia de la filosofía pura sólo sería posible mediante un conocimiento mediato. Pero el conocimiento mediato sólo es posible por el hecho de que lo desconocido se piensa como contenido en algo ya conocido, y le es atribuido el predicado contenido en lo conocido porque lo desconocido está contenido en lo conocido. Pero a un conocimiento que surge de la subsunción de lo particular bajo lo universal lo llamamos un conocimiento mediante inferencias. Por consiguiente, la materia de la filosofía pura sólo es posible mediante inferencias. Por eso cada proposición de la filosofía pura debe estar fundamentada mediante alguna otra, y ésta debe tener su fundamento en una proposición superior, y ésta a su vez en otra superior, etc. Por eso procede de la consecuencia el fundamento, y a su vez asciende desde este fundamento, tomado como consecuencia, hacia otro fundamento superior, 
etc. Mediante este entramado interno y necesario de conocimientos se determina tanto la forma de la ciencia, que consiste en un entramado causal, como también la materia de la misma (el conocimiento).

¿Puede entonces continuar infinitamente nuestro progreso de fundamento en fundamento? ¿No tenemos que llegar a un punto del que después podamos partir, a una proposición que contenga en sí misma el fundamento de todos nuestros fundamentos e inferencias - a un principio fundamental [Grundsat:]? - Y si debemos partir de un primer principio tal, se plantea la siguiente pregunta: ¿puede haber un principio absolutísimo y enteramente incondicionado, que no requiera prueba alguna, que esté fundado en sí mismo, y sin embargo fundamente todas las proposiciones? ¿Un principio tal que ir más allá de él no fuese ni necesario ni posible?

Una proposición puede obligarnos a ir más allá de ella o bien en símisma, o bien con respecto a su consecuencia. En si misma: a fin de probar la conexión del predicado con el sujeto; con respecto su consecuencia: a fin de probar la conexión necesaria de la consecuencia con la proposición. - Por eso en relación con un supremo principio fundamental de toda la filosofía se plantean dos preguntas: ¿puede haber una proposición que en sí misma no requiera prueba alguna? Y también: ¿puede haber una proposición que no tengamos que rebasar por lo que respecta a sus consecuencias?

Si la mente humana (en la medida en que su constitución no nos es conocida por una conciencia inmediata) es objeto de la filosofía pura, pero en la filosofía pura sólo es posible un conocimiento mediante inferencias, entonces la proposición de la que partimos en esta filosofía y de la que queremos hacer el fundamento de todo nuestro filosofar no puede expresar otra cosa que un factum dado en la conciencia y que se refiere a la mente humana. Pues sólo los facta tienen certeza inmediata. Lo que no está dado como factum debe ser probado. Pero como el principio supremo de la filosofía debe expresar algo que sea inmediatamente cierto, no puede expresar otra cosa que un factum. - Por tanto, si el principio que situamos en la cúspide de toda la filosofía expresa algo inmediatamente cierto (sea esto un becho [Tatsache] o una acción [Tathandlung]), no necesitamos ningún principio superior para probarlo; su verdad se sostiene sobre el factum que el principio expresa; y si su contenido está realmente dado en la conciencia, sólo se requiere una reflexión desprejuiciada sobre esa conciencia para encontrarlo verdadero.

Por consiguiente, puede afirmarse que puede haber un principio que en sí mismo no requiera una prueba superior (un juicio que lo fundamente).

"Pero ¿cómo sabemos — podría objetarse- que nuestra reflexión no está equivocada y que la conciencia no nos ha engañado? ¿Cómo nos aseguramos de la corrección de nuestra reflexión? - ¿Repitiendo la reflexión? Pero ésta puede errar 
de nuevo, y cegados por la clara apariencia del primer engaño, podríamos no ver una segunda o una tercera vez lo que veríamos con ojos no cegados. Por tanto, para convencernos y convencer a otros de la estricta certeza del dato que formulamos en nuestro principio, debemos probar nuestro supuesto principio supremo, y por consiguiente buscar un principio superior que lo fundamente. Pero este principio superior tiene que expresar a su vez un factum que entonces, por la misma razones, tiene que ser probado, y así in indefinitum."

Sólo yo puedo convencerme de la corrección de un factum dado en la conciencia. Si el factum está constituido de tal modo que tengo que pensarlo como necesariamente presente [vorhanden]; si está constituido de tal modo que me acompaña en todo representar, que simplemente no soy capaz de eliminarlo con el pensamiento sin suprimirme a mí mismo como sujeto de la representación, entonces no necesito convencerme de la verdad y la necesidad de este factum dado en la conciencia mediante una reflexión repetida o mediante un juicio superior. El criterio más seguro de la verdad de un factum tal consiste en que sencillamente no puedo suprimirlo, ni puedo pensarlo como no existente sin contradecirme a mí mismo.

Ahora bien, a pesar de que, por un lado, creemos tener que defender un principio fundamental absolutísimo de toda la filosofía, nos parece que, por otro lado, no hace falta mucho esfuerzo para comprender que un principio tal es imposible, y que una filosofía completa en sus fundamentos es una mera idea, y que la tarea de la razón que filosofa consiste en avanzar permanentemente hacia principios superiores.

El contenido del principio supremo debe ser algo inmediatamente dado en la conciencia, un factum, y el principio tiene por objetivo trasladarnos al ámbito, desconocido para nosotros, del espíritu humano. Pero ¿cómo es posible esto? - No de otro modo, creo yo, que mediante la relación de lo condicionado con la condición. Pasamos de lo dado en la conciencia como un efecto, a lo que no está dado inmediatamente en la conciencia como causa de lo anterior; del factum en tanto que algo condicionado, a una determinada constitución de la mente humana como su condición. Inferimos así: porque este A nos es dado, debe existir B; pues si no existiese B, no podría existir A. - Pero ¿cómo sabemos que $\mathrm{B}$ es la condición de $\mathrm{A}$ ? ¿Por qué, de entre todas las condiciones posibles, es sólo la condición $\mathrm{B}$ aquella que fundamenta $\mathrm{A}$ ? - Tomemos como ejemplo el principio de conciencia de Rheinhold: la representación es distinguida del sujeto y del objeto por el sujeto, y referida a ambos. Este principio se presenta como un factum de la conciencia en la conciencia; y de él se infiere que algo de la representación debe pertenecer al representante y algo a lo representado, que aquello que en la 
representación pertenece el sujeto es la forma y lo que pertenece al objeto es la materia, etc. Las formas de la mente humana y de la materia dada desde fuera se establecen por tanto como condiciones de este factum. Pero en esa conclusión echo de menos una premisa. ¿Dónde está el fundamento de la conexión de lo condicionado con esta determinada condición? ¿Cómo sé que esta determinada constitución de la mente humana es condición de ese factum? ¿No puede tener ese factum su fundamento en algo diferente? - Que la representación está referida al sujeto representante — podría decir el escéptico— no prueba aún que algo en la representación pertenezca al sujeto. Suponiendo que la materia y la forma fuesen dadas desde fuera, la representación podría, no obstante, ser referida al sujeto. La representación bien puede estar también referida al sujeto porque le es dada a éste y el sujeto es el que la tiene, no simplemente porque el sujeto haya creado la representación. Por consiguiente, ese factum de la conciencia no nos permite inferir con certeza esta condición determinada, o tiene que probarse una proposición que aporte la premisa de esta inferencia y nos demuestre que entre todas las posibles condiciones sólo esta condición podría fundamentar ese factum.

De esta forma de dudar se ha servido también Enesidemo contra Kant. Cuando éste infiere de la necesidad de los juicios su origen a priori, dice Enesidemo (p. 45): "Si las cosas en sí nos son completamente desconocidas, tampoco podemos saber en absoluto cuáles determinaciones de nuestra mente pueden ser producidas por la influencia de las cosas en sí sobre ella, y cuáles no pueden serlo. - Por tanto, ¿por qué debería ser apodícticamente cierto que los objetos reales de las sensaciones, completamente desconocidos para nosotros, no podrían producir conocimientos en los que haya necesidad?" - Por eso Enesidemo reclama para la estricta certeza de esa inferencia una proposición que fundamente la necesidad de la conexión de lo condicionado con la condición determinada, y mediante la cual (y sólo mediante ella) podamos tener la certeza de que no nos hemos equivocado en nuestra inferencia de la condición a lo condicionado. La proposición "es imposible alcanzar la necesidad por medio de la experiencia" no puede ser todavía la proposición buscada. Pues de todas las posibles causas de la necesidad de un juicio, esta proposición sólo excluye una posibilidad. ¿Cuál es la proposición que me demuestre que sólo la mente humana puede ser la única entre todas las posibles causas de este dato?

Así pues, en la medida en que el principio fundamental de toda la filosofía deba expresar un factum a partir del cual se pretenda inferir una determinada condición suya, se presupone siempre un principio superior que nos permita inferir a partir del factum A, como a partir de algo dado y condicionado, a B como su condición, 
y que fundamente la necesidad de la conexión de algo condicionado con una determinada condición.

$\mathrm{Si}$ bien puede ser verdad que $\mathrm{A}$ no requiera prueba alguna porque es un factum, no obstante la relación de este A con B, como su condición, requiere una demostración. Pues esta relación no es inmediatamente cierta, y por tanto debe ser probada. Un principio tal (que expresa la condición de un factum - que propiamente es su fundamento-), establecido como principio fundamental supremo, debe ser pues considerado como una premisa menor y una conclusión sin premisa mayor, y nuestra propia razón nos obliga, por tanto, a seguir ascendiendo en busca de esa premisa mayor. Pero para cada proposición que queramos situar como premisa mayor de esta inferencia se repetirá el caso anterior: o bien no expresa nada inmediatamente dado en la conciencia, y en tal caso se sigue por sí mismo que la proposición debe ser probada, puesto que sólo los facta pueden tener certeza inmediata; o bien la proposición expresa un factum, y entonces sólo puede ser inferida mediante la relación de la condición a lo condicionado. Y así ad infinitum.

Por consiguiente, nunca podemos considerar completo nuestro ascenso a los fundamentos del saber. Nos lo impide la razón misma, que no puede permanecer en una conclusión cuya premisa mayor no está determinada.

A este argumento contra la posibilidad de una filosofía completa en sus fundamentos cabe añadir otro, que refleja el espíritu del criticismo. Es el siguiente, tomado de la teoría kantiana de las ideas.

La razón es la facultad de concebir, como el entendimiento es la facultad de pensar. Esta facultad consiste, pues, en representarse lo fundamentado en conexión con su fundamento, lo condicionado en conexión con su condición. En virtud de esta capacidad, la razón está en posesión de las ideas de lo incondicionado, que pone en la cúspide de lo condicionado como principios de todo concebir. Pero estas ideas no son principios constitutivos, sino meros supuestos de la razón para la regulación de su uso. Así, la idea de la sustancia absoluta es un supuesto de la razón pura en tanto que un principio subjetivo de su investigación, mediante el cual piensa todos los accidentes como fundamentados en un sustrato [Subject] último, que ya no es a su vez accidente sino sólo sustrato. Y mediante este principio se fija la tarea de buscar el objeto anticipado por estas ideas, avanzando hacia él infinitamente o en una serie ascendente indeterminada, y ampliando así la esfera de sus conocimientos. Y otro tanto sucede con todas las ideas de la razón pura.

Todos los conocimientos obtenidos a partir de fundamentos son condicionados. Por eso la razón debe, en virtud de su naturaleza y a fin de regular su uso en la indagación de los fundamentos del conocimiento, pensar toda la serie de 
conocimientos como condicionada mediante un conocimiento incondicionado. Ahora bien, esta idea del conocimiento incondicionado es la idea de la causa absoluta, aplicada a una pluralidad de conocimientos que, como lo incondicionado de los conocimientos condicionados por ella, se llama principio fundamental en la medida en que es pensado como expresado en una proposición; y que debe ser pensado como conocimiento incondicionado, en tanto que no condicionado por nada superior, sino como la clave de bóveda de todo el sistema de los conocimientos condicionados. Pero todas las ideas de lo incondicionado no son más que principios regulativos; principios subjetivos de la razón para la regulación de su uso. Y por tanto, también lo es la idea del conocimiento incondicionado. Mediante esta idea, la razón piensa la serie de los conocimientos condicionados como una serie cerrada, y mediante esta idea, en tanto que supuesto subjetivo, se regula su uso en la indagación de los fundamentos de los conocimientos. En virtud de esta idea debe la razón progresar siempre en la serie de los conocimientos; debe buscar un conocimiento incondicionado como si realmente fuese posible encontrarlo, es decir: debe elevarse de condiciones en condiciones, de fundamentos en fundamentos, en un regreso infinito. - Así pues, así como las ideas de lo incondicionado sólo son un principio regulativo de la razón en la investigación de la naturaleza, así la idea de un fundamento incondicionado es sólo un principio regulativo de la razón en la construcción de la ciencia.

De lo anterior se siguen los siguientes resultados:

1) un principio fundamental incondicionado tiene realidad [Realität] considerado como idea; pero esta idea no puede realizarse nunca y sólo es de uso regulativo. De ahí que

2) en la búsqueda de un principio supremo fundamental, la razón no puede alcanzar nunca su objetivo; no puede llegar nunca en la serie de los conocimientos a un principio incondicionado; - su objetivo es infinito y no puede alcanzarse nunca. Debe progresar siempre hacia la ciencia, y se niega a sí misma si pretende permanecer en un punto como si fuese la última frontera. Avanzar eternamente es la tarea que le impone la idea del conocimiento incondicionado.

3) Mediante la filosofía crítica se torna sencillamente imposible un principio fundamental supremo fundado en sí mismo, y una filosofía que se llama a sí misma filosofía crítica y pretende fundamentarse en un principio supremo y absoluto, se contradice a sí misma y deja de ser filosofía crítica. 
Pero ¿qué sucede entonces con la dignidad y la verdad de la filosofía? ¡Qué clase de ciencia es esa que nunca puede basarse en fundamentos firmes, en la que el espíritu humano nunca alcanza el reposo, y en la que sólo puede luchar, pero no conquistar nada! ¿Con qué derecho confiaríamos a su protección lo más sagrado que conocemos; a ella, que no conoce lugar seguro alguno en el que pudiera arraigar, ni terreno firme alguno que pudiese establecer como fundamento de su edificio?

Por supuesto, a primera vista la existencia o no existencia de la filosofía, y junto con ella el bien o la desgracia de la humanidad, parecen basarse en la existencia o no existencia, en la posibilidad o imposibilidad de un principio fundamental incondicionado. Pero si se investiga la cuestión con detenimiento, el resultado es diferente. - También puede hallarse verdad en la filosofía, aunque ésta no pueda pretender una certeza matemática; la virtud, el derecho, la divinidad y la inmortalidad pueden quedar bajo su protección, y de este modo puede la filosofía realizar su sublime destino, aunque nunca pueda estar completa en sus fundamentos últimos y el progreso bacia la ciencia (en la medida en que se entienda por tal la indagación de un principio incondicionado) nunca pueda finalizarse. Se me permitirá presentar en otra ocasión la prueba de esta afirmación.

Por lo que respecta a la dignidad de la razón que filosofa, por mi parte estoy convencido de que, lejos de ser rebajada, más bien aumenta de este modo. El espíritu se siente elevado por el pensamiento de que la razón que filosofa se eleva de un nivel a otro, y de que tiene un objetivo que, como ella misma, es infinito. 